\title{
MECANISMOS DE CONTROL INTERNO PARA MEJORAR LOS RESULTADOS DE UN PROGRAMA SOCIAL: PROGRAMA JÓVENES A LA OBRA
}

\author{
INTERNAL CONTROL MECHANISMS TO IMPROVE THE RESULTS OF A SOCIAL \\ PROGRAM: PROGRAMA JÓVENES A LA OBRA
}

Diana Almanza Hurtado Universidad Nacional Mayor de San Marcos Lima, Perú

ORCID: https://orcid.org/0000-0001-7554-7834

Correo electrónico: almanza_2@hotmail.com

\section{RESUMEN}

Objetivo: Identificar los mecanismos de control interno que coadyuvan a la mejora de los resultados del Programa Nacional de Empleo Juvenil Jóvenes a la Obra, a través de la ejecución presupuestal, suscripción de convenios y el seguimiento de las tareas programadas. Método: El diseño es no experimental, cualitativo con lo cual se pretende dar respuesta a un fenómeno poco estudiado. Siendo una investigación del tipo descriptivo - explicativo, se elaboraron encuestas para recaudar información pertinente al tema en las que se utilizó la escala de Likert. La recaudación de datos se obtuvo de una población de 80 personas del Programa Jóvenes a la Obra, que laboraron durante el periodo 2014, y para la muestra se utilizó el método del muestreo no probabilístico. Resultados: La implementación de mecanismos de control interno mejorarán los resultados del Programa, optimizando la gestión del mismo. Conclusisón: Los mecanismos de control interno en el marco de la ejecución presupuestal, ejecución de convenios y seguimiento de tareas programadas coadyuvaran a la mejora de la gestión del Programa Jóvenes a la Obra, logrando el incremento de la inserción laboral de los beneficiarios.

Palabras clave: Control; convenio; suscripción; ejecución; optimización.

\begin{abstract}
Objective: To identify the internal control mechanisms that contribute to the improvement of the results of the National Youth Employment Program Young People, through a budgetary execution, signing of agreements and the monitoring of the programmed tasks. Method: The non - experimental design was used, qualitative in which pretend to respond to a little studied phenomenon. Being a research type exploratory - descriptive, it was elaborated surveys to collect relevant information to the subject in which it was use the Likert scale. The data collection was obtained from a population of 80 people of the youth to the work program, who worked during the 2014 period and for the sample the method of non-probabilistic sampling was used. Results: The implementation of internal control mechanisms will improve the results of the Youth to Work Program while optimizing its management. Conclusions: The mechanisms of internal control within the framework of budgetary execution, execution of agreements and follow-up of scheduled tasks will contribute to the improvement of the management of the Youth to Work Program, attaining an increase in the labor insertion of the beneficiaries.
\end{abstract}

Keywords: Control; agreement; subscription; execution; optimization.

(c) Los autores. Este artículo es publicado por la Revista Quipukamayoc, Universidad Nacional Mayor de San Marcos. Este es un artículo de acceso abierto, distribuido bajo los términos de la Licencia Creative Commons Atribución-NoComercial-Compartirlgual 4.0 Internacional.(http://creativecommons.org/licenses/by-nc-sa/4.0/), que permite el uso no comercial, distribución y reproducción en cualquier medio, siempre que la obra original sea debidamente citadas. 


\section{INTRODUCCIÓN}

El Programa Nacional de Empleo Juvenil Jóvenes a la Obra, en adelante denominado como Programa, fue creado mediante Decreto Supremo N ${ }^{\circ}$ 013-2011-TRABAJO de 20 de agosto de 2011, siendo constituida como una Unidad Ejecutora perteneciente al Ministerio de Trabajo y financiado con recursos del Tesoro Público, donaciones, cooperación técnica internacional y otras fuentes de financiamiento.

El Programa tiene el propósito de promover la empleabilidad de los jóvenes en ocupaciones de calificación inicial siendo el eje central la capacitación a través de cursos de alta calidad con el objetivo de facilitar el acceso al mercado laboral a jóvenes de escasos recursos económicos, a través de acciones específicas de capacitación técnica, experiencia laboral y otras modalidades de capacitación demandadas por el mercado laboral.

El Programa institucionaliza y gestiona con autonomía los intereses propios de las correspondientes colectividades; siendo elementos esenciales la formación laboral y la inserción al mercado laboral. Así pues, el Programa brinda información referida a las ocupaciones que el mercado demanda, principalmente para sectores como el agropecuario, comercio, construcción, industria, pesca, servicios, transporte, almacenamiento y comunicaciones.

Durante el proceso del desarrollo de las capacitaciones a los beneficiarios se han observado hechos que habrían motivado un incremento en los niveles de deserción de los beneficiarios, lo cual afectaría el objetivo del Programa causado por (a) el cambio de docentes, horarios y locales de las capacitaciones; (b) modificación de las fechas de inicio termino de los cursos de capacitación y (c) puestos laborales insuficientes.

Por lo que, los mecanismos de control interno del Programa se realizaron a partir de la hipótesis general siguiente: Los mecanismos de control interno contribuyen a mejorar los resultados del Programa Jóvenes a la Obra.

El Programa no ha contado con un sistema de monitoreo de la ejecución de los convenios, ya que, en todos los casos, la información de la ejecución de la capacitación corría a cargo de las Entidades de Capacitación (en adelante ECAP). Incluso para los docentes se espera la contratación de los mismos en cada provincia a atender, pero no hay evidencia de que se haya diseñado un plan para la contratación de los mismos, más aún cuando en determinadas provincias no se cuenta con personal del perfil que se requiere para la labor.
Asimismo, el Ministerio de Trabajo (2012) señala que los factores que incidieron negativamente en el cumplimiento de las metas y objetivos son:

- Demora por parte del Ministerio de Economía y Finanzas (MEF) en la consulta y validación de elegibilidad de jóvenes acreditados en el Sistema de Focalización de Hogares (SISFOH).

- Demora por parte de las entidades de capacitación en absolver observaciones y/o sugerencias efectuadas por la Unidad Técnica Operativa sobre sus propuestas técnicas presentadas. Las observaciones recurrentes estuvieron centradas en los sobrecostos de las propuestas; esta situación contribuyó a que se dilate el proceso de autorización de inicio de curso.

- Demora por parte de la Oficina General de Planeamiento y Presupuesto del Ministerio de Trabajo y Promoción del Empleo en la autorización de modificaciones presupuestales; generó retrasos en la adquisición de bienes y servicios orientados a la operatividad de las intervenciones del programa.

- Limitada capacidad operativa de las entidades de capacitación para brindar sus servicios a partir del mes de marzo, debido a que en este mes empiezan sus labores de formación cotidianas.

Otra consecuencia generada de los factores que inciden negativamente en el cumplimiento de metas es el menor número de empresas interesadas en contratar a los beneficiarios, debido a los retrasos en la culminación de la capacitación que no coinciden con las fechas de demanda de empleo para las empresas y en la menor oferta de potenciales trabajadores debido a los niveles de deserción.

Como se ha mencionado, el objetivo del Programa es contribuir a la formación laboral e inserción en el mercado laboral. Para ello requiere ejecutar programas de capacitación bajo la modalidad de Presupuesto por Resultados (en adelante PpR).

En resumen, se ha observado dificultades en la ejecución de las capacitaciones que han incidido en los niveles de deserción, así como en una menor ejecución presupuestal. Por lo tanto, esta investigación pretende mostrar el rol de los mecanismos de control interno para optimizar la gestión del Programa.

El control de la ejecución de programas en el Estado peruano es un tema de investigación escasamente desarrollado. Así pues, de acuerdo al Centro Nacional de Planeamiento Estratégico (2012) (en adelante Ceplan), entre las características principales acerca del diagnóstico de los programas presupuestales y su seguimiento se tiene: 
a. Escasa claridad acerca de la relación entre los objetivos del gasto público y las prioridades de la política.

b. Escasa información acerca del diseño de las intervenciones públicas, así como de su desempeño.

c. Escasa cultura de evaluación en el sector público.

d. Escasa rendición de cuentas.

Por otro lado, la literatura referida al estudio del control interno o sistemas de control como herramientas para la mejora de la gestión pública está concentrada principalmente en el concepto de control interno definido en la Ley 28716, Ley de Control Interno de las Entidades del Estado; así como en la Ley 27785, Ley Orgánica del Sistema Nacional de Control y de la Contraloría General de La República.

No obstante, la escasez de investigaciones al respecto se puede observar que existen estudios que abordan el problema de control de gestión basados en modelos de PpR, los cuales recomiendan que los programas que se ejecuten bajo este modelo implementen una cultura de planificación y metas a través de instrumentos como el Marco Lógico, y que las instituciones deben previamente establecer sus objetivos, así como las metas y actividades asociadas al cumplimiento de dichos objetivos (Contraloría General de la República, 2008).

Aun cuando se identifica la importancia de los objetivos, metas y actividades asociadas, estos son conceptos que se entienden de distintas maneras.

Leiva y Soto (2015) señalan que:

No existe consenso en la definición de objetivos nacionales ni sectoriales; $y$ peor aún en la determinación de brechas que se debe cubrir como país. Los sectores y los gobiernos regionales y locales definen aún sus prioridades sin considerar las prioridades nacionales y a su vez el gobierno nacional define sus objetivos sin considerar las prioridades locales, lo que redunda en el poco alineamiento de politicas y estrategias ( $p$. 12).

En cuanto al control de los objetivos, Starling (2011) indica que:

Es necesario un conjunto de herramientas para la gestión pública que permitan coadyuvar a resolver los problemas de ésta, caracterizados por la colaboración y los retos que supone cumplir con los objetivos en este tipo de contextos colaborativos (p. 550).

Salamón (2012) señala que:

En la implementación de programas en la gestión pública requiere de herramientas que faciliten lo- grar: (b) Efectividad; (c) Eficiencia; (d) Equidad (p. 1647).

Este autor refiere que algunas de estas herramientas provienen de la Gestión de la Calidad Total, principalmente aquellas que buscan la mejora continua como los diagramas de causa-efecto, estudios estadísticos correlacionales (gráficas de dispersión), y gráficas de control.

De acuerdo con Cejudo y Maldonado (2011), los sistemas de control y monitoreo pueden tener muchos propósitos vinculados con los procesos internos, que incluyen:

(a) control presupuestal; (b) desarrollo de mecanismos de seguimiento a la gestión; (c) control del desempeño de funcionarios o agencias externos a él. En todos los casos, estos sistemas buscan mejorar el desempeño de los programas. No obstante, estos múltiples usos plantean un problema ya que no están definidas las tipologías de los sistemas de control, o qué elementos lo componen para cada tipo de programa (p. 396).

En años recientes, específicamente desde el año 2006 empieza a utilizarse en el Perú el PpR, como herramienta en la que ayuda a mejorar: a) la asignación y uso de recursos públicos b) calidad del gasto a través del fortalecimiento de la relación entre presupuesto y resultado y c) demanda de un uso más intenso de información para la medición del desempeño (Centro Nacional de Planeamiento Estratégico, 2012).

El PpR es un instrumento que vincula los resultados, a través de los productos (bienes y servicios) que se generan tras una intervención dentro de un programa presupuestal con los recursos asignados a ese programa. Este instrumento facilita el seguimiento al gasto en los programas (ejecución financiera y comparación de certificaciones, compromisos y devengue), entrega de productos e indicadores de desempeño de resultados a partir de los productos entregados (cuyo monitoreo corresponde al Instituto Nacional de Estadística e Informática - INEI). No obstante, la metodología de seguimiento, de acuerdo con el Ceplan, implica monitoreos trimestrales con contraste de data en el Sistema de Integración Financiera (SIAF), y de mayor plazo a través de resultados de encuestas aplicadas por INEI (Encuesta Nacional de Hogares sobre condiciones de Vida y Pobreza, Encuesta Nacional a Instituciones Educativas, entre otras). Esta periodicidad de tres meses a un año, para la comparación de los datos y la toma de medidas correctivas no siempre es suficiente para programas de inversión pública como Jóvenes a la Obra.

Asimismo, el enfoque hacia resultados y recursos pierde de vista actividades intermedias que contribuyen además en la implementación exitosa de los programas, como el 
seguimiento a la ejecución de los plazos para las contrataciones por demoras en expedición de términos de referencia, trabas administrativas, incumplimiento de condiciones pactadas por proveedores, entre otros.

El Programa Jóvenes tiene como propósito mejorar las condiciones de empleabilidad de jóvenes de 18 a 29 años de edad. De acuerdo al Ministerio de Trabajo y Promoción del Empleo, este programa cuenta con dos líneas de acción: (a) Capacitación para la inserción laboral y (b) Capacitación para el emprendimiento, en el que se incluye la capacitación y asistencia técnica para la formulación del plan de negocios. Para la primera línea de acción se han firmado convenios con empresas privadas para que acojan a los jóvenes beneficiados. Los empleos en los que se insertan laboralmente los jóvenes beneficiados incluyen oficios como embalador a mano, embalador de cajas y obrero de productos terminados, entre otros.

El programa brinda la capacitación a través de entidades de capacitación como universidades e institutos. Los cursos que se incluyen en la formación están orientadas a cubrir la demanda, o en donde se observa mayores posibilidades de inserción, entre los cursos se incluyen: (a) confección textil, (b) operarios de producción, (c) operatividad de máquinas industriales, (d) logística y almacenes, (e) telemarketing, (f) mecánica y electricidad automotriz, (g) construcción, (h) cocina, (i) panadería y pastelería, (j) ventas.

Asimismo, durante el periodo agosto 2011 - diciembre 2013 el programa ha realizado una ejecución presupuestal de PEN 84,8 millones, beneficiando a 54 mil jóvenes identificados como pobres o pobres extremos (Ministerio de Trabajo y Promoción del Empleo, 2014).

Cabe señalar que el Programa cuenta con un sistema de monitoreo que mide tres aspectos, fundamentalmente: (a) el de los propios jóvenes usando la base de datos de postulantes/participantes; (b) el de las entidades capacitadoras y (c) el de los capacitados luego de la fase de capacitación (Contraloría General de la República, 2008).

Asimismo, el Grupo de Análisis para el Desarrollo (GRADE) hizo una evaluación del impacto de este programa, siendo los resultados para GRADE (2005) los siguientes:

- Los participantes obtuvieron un ingreso por hora $33 \%$ superior al grupo de control.

- Los participantes tenían una probabilidad mayor entre $4 \%$ a $7 \%$ a ser empleados que el grupo de control. Sin embargo, la desviación estándar para este indicador resultó siendo muy alta, por lo que no se considera significativo.
- Para aquellos participantes que, si consiguieron empleo, las horas laboradas eran mayores que para el grupo de control.

Pese a estos resultados, se han observado deficiencias en la ejecución del programa, como se ha descrito en el apartado 1.1 referidos a la ejecución de la capacitación y ejecución presupuestal. Más aún, no existe un cuerpo homogéneo de herramientas preestablecidas para realizar el seguimiento de la ejecución de programas como Jóvenes a la Obra.

Los mecanismos de control interno contribuyen a mejorar los resultados del Programa Jóvenes a la Obra.

a. Los mecanismos de control interno permiten el seguimiento de la ejecución presupuestal.

b. Los mecanismos de control interno permiten el seguimiento de control de convenios.

c. Los mecanismos de control interno permiten el seguimiento de las tareas programadas.

Sobre la justificación teórica, la revisión de la literatura muestra que existen definiciones imprecisas respecto a los conceptos que conforman el objeto de estudio. Así pues, respecto al control de gestión en el sector público, éste en algunos casos es vinculado a procesos legales en el que los órganos de control interno juegan un rol rector y en el que se fija la atención en el cumplimiento de procesos normados legalmente para evitar la corrupción en distintas instancias del aparato estatal. Por otro lado, existe una visión económica de la gestión pública que principalmente está vinculada al marco lógico del PpR.

Ante ambas perspectivas, cabe precisar si estas definiciones son apropiadas para a su vez definir los procedimientos de control interno que permitan optimizar y llevar a cabo una gestión exitosa en programas como "Jóvenes a la Obra”. En ese sentido, una primera justificación de la investigación se presenta en coadyuvar en definir conceptos no explicados en la literatura aplicados en la gestión de programas presupuestales.

En cuanto a, la justificación práctica, la investigación presentada pretende ser un elemento de guía y utilidad para la aplicación e implementación de medidas de control interno en el Programa Jóvenes a la Obra.

Asimismo, pretende servir como instrumento de verificación de la ejecución presupuestal y de inserción laboral como expresión de los objetivos y metas trazadas por la entidad, la cual direcciona recursos previstos para la ejecución de los convenios y el cumplimiento de la visión y misión del programa. 
También serviría como herramienta de ejecución de políticas públicas para atender las demandas y necesidades de la sociedad, a través del control del gasto público y el nivel de inserción laboral.

\section{MATERIAL Y MÉTODOS}

La investigación empleó el diseño no experimental, cualitativo con lo cual se pretende dar respuesta a un fenómeno poco estudiado y en el que se pretende dar respuesta a preguntas acerca de un problema escasamente estudiado y/o profundizado. Siendo una investigación del tipo descriptivo - explicativo, se elaboraron encuestas para recaudar información pertinente al tema en las que se utilizó la escala de Likert. La recaudación de datos se obtuvo de una población de 80 personas del Programa Jóvenes a la Obra, que laboraron durante el periodo 2014. Para la muestra se utilizó el muestreo intencionado y no probabilístico, en el cual el investigador selecciona los elementos que serán representativos, procediéndose a realizar la recolección de datos a 33 personas que laboraron en el Programa Jóvenes a la Obra durante el año 2014.

\section{RESULTADOS}

Según un análisis descriptivo de la recolección de datos, se obtuvo como resultado que, de las 33 encuestas aplicadas a trabajadores del Programa Jóvenes a la Obra, el 77\% está de acuerdo con la hipótesis general, el 76\% está de acuerdo con la primera hipótesis específica, el 73\% están de acuerdo con la segunda hipótesis específica y por último el $68 \%$ está de acuerdo con la tercera hipótesis específica, tal como se detalla en la Tabla 1.
Los mecanismos de control interno permiten el seguimiento de la ejecución presupuestal.

Contribuye a la mejora en la medición de la certificación, compromiso y devengado respecto al marco presupuestal, hecho que mejoraría los resultados del programa Jóvenes a la Obra.

Ho: Los mecanismos de control interno no permiten el seguimiento de la ejecución presupuestal.

$\mathrm{H}_{1}$. Los mecanismos de control interno permiten el seguimiento de la ejecución presupuestal.

Con los datos recaudados en la investigación se procede a realizar la prueba de chi-cuadrado tal como se puede observar en la Tabla 2.

Se obtiene como resultado que el valor de $\mathrm{P}(0,000)$ es < que a $(0,05)$ por lo que rechazamos la hipótesis nula Ho y aceptamos la hipótesis alterna $\mathrm{H}_{1}$, las variables mecanismos de control interno permiten el seguimiento de la ejecución presupuestal SI ESTAN ASOCIADAS, con un nivel de significancia del 95\% y con 1 grados de libertad (Ver tabla 3).

De la elaboración de las medidas simétricas para primera hipótesis específica, se tiene como resultado que el coeficiente de contingencia de Pearson tiene un valor de 0.51 lo que nos indica que existe una relación positiva, directa y que esta relación es alta.

Los mecanismos de control interno permiten el seguimiento de control de convenios.

Tabla 1

Resultado de las encuestas en porcentajes

\begin{tabular}{|c|c|c|c|}
\hline Hipótesis & Descripción & Frecuencias & De acuerdo \\
\hline Hipótesis General & Los mecanismos de control interno & 228 & $77 \%$ \\
\hline Primera Hipótesis Específica & Seguimiento de la ejecución presupuestal & 225 & $76 \%$ \\
\hline Segunda Hipótesis Específica & Seguimiento de control de convenios & 216 & $73 \%$ \\
\hline Tercera Hipótesis Específica & Seguimiento de las tareas programadas & 201 & $68 \%$ \\
\hline
\end{tabular}

Fuente. Elaboración Propia

Tabla 2

Prueba de chi-cuadrado para la primera hipótesis especifica

\begin{tabular}{lccc}
\hline Detalle & Valor & Grado de Libertad & Sig. asintótica (2 caras) \\
\hline Chi-cuadrado de Pearson & 12,186 & 1 & 0,000 \\
\hline
\end{tabular}

Fuente. Elaboración Propia 
Facilitan realizar las verificaciones mensuales de las actividades de capacitación, cantidad de matriculados, contratación oportuna de servicios, entre otros, mejorando los resultados del programa Jóvenes a la Obra.

Ho: Los mecanismos de control interno no permiten el seguimiento de control de convenios.

$\mathrm{H}_{1:}$ Los mecanismos de control interno permiten el seguimiento de control de convenios.

La prueba de chi-cuadrado para la segunda hipótesis específica se puede visualizar en la Tabla 4.

Según las medidas simétricas para la segunda hipótesis específica se obtuvo como resultado que el valor de $\mathrm{P}(0,003)$ es $<$ que $\alpha(0,05)$ por lo que rechazamos la hipótesis nula Ho y aceptamos la hipótesis alterna $\mathrm{H} 1$ las variables los mecanismos de control interno permiten el seguimiento de control de convenios, SI ESTÁN ASOCIADAS, con un nivel de significancia del 95\% y con 1 grados de libertad, esto se ve reflejado en la Tabla 5.

De la elaboración de las medidas simétricas, para la segunda hipótesis específica, se obtuvo que el coeficiente de contingencia de Pearson tiene un valor de 0.46 lo que nos indica que existe una relación positiva y directa entre las dos variables y que esta relación es Moderada.

Los mecanismos de control interno permiten el seguimiento de las tareas programadas.

Contribuyen a mejorar los resultados del Programa Jóvenes a la Obra.

Ho: Los mecanismos de control interno no permiten el seguimiento de las tareas programadas.

H1: Los mecanismos de control interno permiten el seguimiento de las tareas programadas.

La prueba de chi-cuadrado para la tercera hipótesis específica se muestra en la Tabla 6.

Si mostramos las medidas simétricas para la tercera hipótesis específica obtenemos como resultado que el valor de $\mathrm{P}(0,000)$ es < que a $(0,05)$ por lo que rechazamos la hipótesis nula Ho y aceptamos la hipótesis alterna $\mathrm{H}_{1}$ las variables los mecanismos de control interno permiten el seguimiento de las tareas programadas, SI ESTÁN ASOCIADAS, con un nivel de significancia del 95\% y con 1 grados de libertad, tal como observamos en la Tabla 7 .

Tabla 3

Medidas simétricas para la primera hipótesis específica

\begin{tabular}{lllc}
\hline & Detalle & Valor & Aprox. Sig. \\
\hline \multirow{3}{*}{ Nominal por Nominal } & Phi & 0,608 & 0,000 \\
& V de Cramer & 0,608 & 0,000 \\
& Coeficiente de contingencia & 0,519 & 0,000 \\
\hline
\end{tabular}

$\mathrm{N}^{\circ}$ de casos válidos

33

Fuente. Elaboración Propia

Tabla 4

Prueba de chi-cuadrado para la segunda hipótesis específica

\begin{tabular}{cccc}
\hline Detalle & Valor & Grado de Libertad & Sig. asintótica (2 caras) \\
\hline Chi-cuadrado de Pearson & 9,066 & 1 & 0,003 \\
\hline
\end{tabular}

Fuente. Elaboración Propia

Tabla 5

Medidas simétricas para la segunda hipótesis específica

\begin{tabular}{|c|c|c|c|}
\hline Detalle & & Valor & Aprox. Sig. \\
\hline \multirow{3}{*}{ Nominal por Nominal } & Phi & 0,524 & 0,003 \\
\hline & V de Cramer & 0,524 & 0,003 \\
\hline & Coeficiente de contingencia & 0,464 & 0,003 \\
\hline
\end{tabular}

$\mathrm{N}^{\circ}$ de casos válidos

33

Fuente. Elaboración Propia 
De la elaboración de las medidas simétricas, para la tercera hipótesis específica se tiene que el coeficiente de contingencia de Pearson tiene un valor de 0,51 lo que nos indica que existe una relación positiva y directa entre las dos variables y que esta relación es Alta.

Ho: Los mecanismos de control interno no permiten la mejora de los resultados del Programa Jóvenes a la Obra.

$\mathrm{H}_{1:}$ Los mecanismos de control interno permiten la mejora de los resultados del Programa Jóvenes a la Obra.

En cuanto a la prueba de chi-cuadrado para resultados del Programa Jóvenes a la Obra se puede observar en la Tabla 8.

Los mecanismos de control interno contribuyen a mejorar los resultados del Programa Jóvenes a la Obra se obtuvo como resultado que el $\mathrm{P}$ valor $(0,003)$ es $<$ que a $(0,05)$ por lo que rechazamos la hipótesis nula Ho y aceptamos la hipótesis alterna $\mathrm{H} 1$ las variables los mecanismos de control interno permiten la mejora de los resultados del Programa Jóvenes a la Obra, SI ESTÁN ASOCIADAS, con un nivel de significancia del 95\% y con 1 grados de libertad (Ver tabla 9).

En relación a las medidas simétricas, para la hipótesis general el coeficiente de contingencia de Pearson tiene un valor de 0,46 lo que nos indica que existe una relación positiva y directa entre las dos variables y que esta relación es Moderada.

\section{DISCUSIÓN}

No existen antecedentes directamente relacionados con las variables de estudio, ya que existe una escasa investigación a los programas sociales. Por tal motivo, esta

Tabla 6

Prueba de chi-cuadrado para la tercera hipótesis específica

\begin{tabular}{lccc}
\hline Detalle & Valor & Grado de Libertad & Sig. asintótica (2 caras) \\
\hline Chi-cuadrado de Pearson & 12,186 & 1 & 0,000 \\
\hline Fuente. Elaboración Propia & &
\end{tabular}

Tabla 7

Medidas simétricas para la tercera hipótesis específica

\begin{tabular}{lllc}
\hline Detalle & & Valor & Aprox. Sig. \\
\cline { 1 - 2 } Nominal por Nominal & V de Cramer & 0,608 & 0,000 \\
& Coeficiente de contingencia & 0,608 & 0,000 \\
$\mathrm{~N}^{\circ}$ de casos válidos & & 0,519 & 0,000 \\
\hline
\end{tabular}

Fuente. Elaboración Propia

Tabla 8

Prueba de chi-cuadrado para resultados del Programa Jóvenes a la Obra

\begin{tabular}{llll}
\hline Detalle & Valor & Grado de Libertad & Sig. asintótica (2 caras) \\
\hline Chi-cuadrado de Pearson & 9,066 & 1 & 0,003 \\
\hline
\end{tabular}

Fuente. Elaboración Propia

Tabla 9

Medidas simétricas para la tercera hipótesis específica

\begin{tabular}{llcc}
\hline Detalle & & Valor & Aprox. Sig. \\
\hline \multirow{2}{*}{ Nominal por Nominal } & Phi & 0,524 & 0,003 \\
& C de Cramer & 0,524 & 0,003 \\
& Coeficiente de contingencia & 0,464 & 0,003 \\
\hline N de casos válidos & & 33 &
\end{tabular}

Fuente. Elaboración Propia 
investigación es muy importante, puesto que, se orienta al control interno y los resultados que se obtendrían en el Programa. Es así que, los resultados emergentes de los mecanismos de control interno permitirán el seguimiento de la ejecución presupuestal, seguimiento de control de convenios y el seguimiento de las tareas programadas lo cual contribuiría a mejorar los resultados del Programa Jóvenes a la Obra. En ese entender al aplicar los mecanismos de control interno para la ejecución presupuestal conllevaría al mejoramiento del presupuesto ejecutado generando un incremento presupuestal en la suscripción de convenios, con ello la ejecución de los mismos y la posibilidad de insertar laboralmente a más beneficiarios. Por lo que, el adecuado seguimiento a las tareas programadas, identificaría de mejor manera el problema durante el proceso de la suscripción y ejecución del convenio. Es así que, el 77\% de los encuestados opina que los mecanismos de control interno mejoraran los resultados del Programa Jóvenes a la Obra, siendo estas dos variables asociadas con un nivel de significancia del $95 \%$, por lo que, existe una relación positiva y directa. En ese entender, de la investigación realizada se obtuvo como resultado que los mecanismos de control interno servirán para mejorar los resultados del Programa Jóvenes a la Obra. Es así que coincidimos con lo investigado por Leiva y Soto (2015), donde el control interno en la Entidad se realiza con el fin de que la gestión de sus recursos, bienes y operaciones se efectúe correcta, eficaz y eficientemente, coadyuvando al logro de los objetivos y misión institucional, así mismo también señalan que en la actualidad existe una serie de problemáticas por la falta de control interno en los procesos internos de una Entidad, siendo parte de ello el desconocimiento de esta, por lo que, el inicio de una implementación de control interno es altamente recomendable de tal forma que se busque a partir de las estrategias/objetivos planteados, a fin de orientar como punto de partida el desarrollo y madurez de la gestión, de modo que se pueda ir avanzando hacia un nivel óptimo o de mejora continua. En ese entender el control interno coadyuva a la mejora continua de toda Entidad, de tal forma que los resultados que se obtienen contribuyen al cumplimiento de sus metas y objetivos, orientando a la implementación de mecanismos de control interno que les sean más idóneas.

\section{REFERENCIAS BIBLIOGRÁFICAS}

Cejudo, G., \& Maldonado, C. (2011). De las recomendaciones a las acciones. Lima: CIDE - SFP.

Centro Nacional de Planeamiento Estratégico. (2012). Instrumentos del PpR: Programas presupuestales, seguimiento, evaluaciones independientes e incentivos. Recuperado de: https://www.ceplan.gob.pe/

Contraloría General de la República. (2008). Programas Sociales en el Perú: Elementos para una propuesta desde el control gubernamental. Recuperado de:

http://www2.congreso.gob.pe/sicr/cendocbib/con2_uibd. nsf/18725BB8EE53C8360525784E006C6812/\$FILE/ programas-sociales_1222469649[1].pdf

Leiva, D., \& Soto, H. (2015). Control interno como herramienta para una gestión pública eficiente y prevención de irregularidades. Lima: CIES.

Ministerio de Trabajo y Promoción del Empleo. (2011) Decreto Supremo $N^{\circ}$ 013-2011-TRABAJO de 20 de agosto de 2011. Recuperado de: http://www.jovenesproductivos.gob.pe/transparencia/DS_013-2011-TR.pdf

Ministerio de Trabajo y Promoción del Empleo. (2018) Mecánica Operativa de los Procesos del Área de Calificación y Selección de Entidades de Capacitación. Recuperado de: http://www.jovenesproductivos.gob.pe/transparencia/resoluciones/rde/2018/RDE104-2018.PDF

Ministerio de Trabajo. (2012). Tercer informe bimensual de ejecución de actividades orientadas a mejorar la empleabilidad de los jóvenes. Recuperado de: http:// www.mintra.gob.pe/archivos/file/informe_bimensual_jovenes_obra.pdf

Salamón, L. M. (2012). The new governance and the tools of the public action: an introduction. In L. M. Salamón (Ed.) The tools of government: The guide to the new governance. New York, NY: Oxford Press.

Starling, G. (2011). Managing the Public Sector (9na. ed.). Boston: Cengage. 